\title{
SOME DEFINITE INTEGRALS ASSOCIATED WITH THE RIEMANN ZETA FUNCTION
}

\author{
H.M. Srivastava \\ Department of Mathematics and Statistics \\ University of Victoria \\ Victoria, British Columbia V8W 3P4, Canada \\ E-Mail: harimsri@math.uvic.ca \\ M.L. Glasser \\ Department of Physics \\ Clarkson University \\ Potsdam, New York 13699-5820, U.S.A. \\ E-Mail: laryg@clarkson.edu \\ and \\ Victor S. Adamchik \\ Department of Applied Computer Science \\ Illinois State University \\ Normal, Illinois 61790-5150, U.S.A. \\ E-Mail: vsadamc@ilstu.edu
}

\begin{abstract}
The authors aim at deriving a family of series representations for $\zeta(2 n+1)(n \in \mathbb{N})$ by evaluating certain trigonometric integrals in several different ways. They also show how the results presented in this paper relate to those that were obtained in other works. Finally, some illustrative computational examples, using Mathematica (Version 4.0) for Linux, are considered.
\end{abstract}

\section{Introduction}

The Riemann Zeta function $\zeta(s)$ and the Hurwitz (generalized) Zeta function $\zeta(s, a)$, which are defined usually by

$$
\zeta(s):= \begin{cases}\sum_{n=1}^{\infty} \frac{1}{n^{s}}=\frac{1}{1-2^{-s}} \sum_{n=1}^{\infty} \frac{1}{(2 n-1)^{s}} & (\mathfrak{R}(s)>1) \\ \frac{1}{1-2^{1-s}} \sum_{n=1}^{\infty} \frac{(-1)^{n-1}}{n^{s}} & (\mathfrak{R}(s)>0 ; s \neq 1)\end{cases}
$$

2000 Mathematics Subject Classification. Primary 11M06; Secondary 11M35, 42A32.

Key words and phrases. Zeta functions, trigonometric integrals, Clausen functions, series representations, trigonometric sums, Mellin transforms. 
and

$$
\zeta(s, a):=\sum_{n=0}^{\infty} \frac{1}{(n+a)^{s}} \quad(\Re(s)>1 ; \quad a \neq 0,-1,-2, \ldots),
$$

can indeed be extended meromorphically to the whole complex $s$-plane except for a simple pole at $s=1$ with residue 1 (see, for details, Titchmarsh [23]). It is easily seen from (1.1) and (1.2) that

$$
\zeta(s, 1)=\zeta(s)=\left(2^{s}-1\right)^{-1} \zeta\left(s, \frac{1}{2}\right) \quad \text { and } \quad \zeta(s, 2)=\zeta(s)-1 .
$$

In recent years there has been a renewed interest in representing $\zeta(2 n+1)(n \in \mathbb{N}), \mathbb{N}$ being the set of positive integers, by menas of series which converge more rapidly than the defining series in (1.1). These developments seem to have stemmed from the use of the familiar series representation (see, e.g., Hjortnaes [14] and Gosper [11]):

$$
\zeta(3)=\frac{5}{2} \sum_{k=1}^{\infty} \frac{(-1)^{k-1}}{k^{3}\left(\begin{array}{c}
2 k \\
k
\end{array}\right)}
$$

in Apéry's proof [2] of the irrationality of $\zeta(3)$, as well as from Ewell's yet another rediscovery [8] of Euler's formula (see, e.g., Ayoub [3, pp. 1084-1085]; see also Ramaswami [19] and Srivastava [20, p. 7, Equation (2.23)]):

$$
\zeta(3)=-\frac{4 \pi^{2}}{7} \sum_{k=0}^{\infty} \frac{\zeta(2 k)}{(2 k+1)(2 k+2) 2^{2 k}} .
$$

The works of (among others) Ewell [9], Dạbrowski [7], Zhang and Williams [24], Cvijović and Klinowski [6], and Srivastava ([21] and [22]) may be cited in connection with the aforementioned developments.

The main object of this paper is to derive a family of series representations for $\zeta(2 n+1)(n \in \mathbb{N})$ by evaluating certain trigonometric integrals in several different ways and to show how the results presented here relate to those that were obtained in other works. We also consider some illustrative computational examples by using Mathematica (Version 4.0) for Linux.

\section{A Cosecant Integral and Its Consequences}

We begin by considering the cosecant integral:

$$
\mathcal{I}_{s}(\omega):=\int_{0}^{\pi / \omega} t^{s} \csc ^{2} t d t \quad(\Re(s)>1 ; \quad \omega>1),
$$

which, upon integration by parts, readily yields

$$
\begin{gathered}
\mathcal{I}_{s}(\omega)=-\left(\frac{\pi}{\omega}\right)^{s} \cot \left(\frac{\pi}{\omega}\right)+s \int_{0}^{\pi / \omega} t^{s-1} \cot t d t \\
(\Re(s)>1 ; \quad \omega>1) .
\end{gathered}
$$


The cotangent integral in (2.2) can, in fact, be evaluated in several different ways. First of all, since (cf., e.g., Magnus et al. [17, p. 36])

$$
z \cot z=-2 \sum_{k=0}^{\infty} \zeta(2 k)\left(\frac{z}{\pi}\right)^{2 k} \quad(|z|<\pi),
$$

we find from (2.2) that

$$
\begin{gathered}
\mathcal{I}_{s}(\omega)=-\left(\frac{\pi}{\omega}\right)^{s} \cot \left(\frac{\pi}{\omega}\right)-2 s\left(\frac{\pi}{\omega}\right)^{s-1} \sum_{k=0}^{\infty} \frac{\zeta(2 k)}{(s+2 k-1) \omega^{2 k}} \\
(\Re(s)>1 ; \quad \omega>1) .
\end{gathered}
$$

In its special case when $\omega=2$, the integral formula (2.4) reduces immediately to the form:

$$
\begin{gathered}
\int_{0}^{\pi / 2} t^{s} \csc ^{2} t d t=-2 s\left(\frac{\pi}{2}\right)^{s-1} \sum_{k=0}^{\infty} \frac{\zeta(2 k)}{(s+2 k-1) 2^{2 k}} \\
(\mathfrak{R}(s)>1),
\end{gathered}
$$

which, for $s \in \mathbb{N} \backslash\{1\}$, appears in the works of (for example) Glasser [10, p. 446, Equation (11)] and Prudnikov et al. [18, p. 388, Entry (2.5.4.6)] (see also Gradshteyn and Ryzhik [12, p. 418, Entry $3.748(2)])$. Furthermore, if we set $\omega=4$ in (2.4), we shall readily obtain the special case:

$$
\begin{gathered}
\int_{0}^{\pi / 4} t^{s} \csc ^{2} t d t=-\left(\frac{\pi}{4}\right)^{s}-2 s\left(\frac{\pi}{4}\right)^{s-1} \sum_{k=0}^{\infty} \frac{\zeta(2 k)}{(s+2 k-1) 4^{2 k}} \\
(\Re(s)>1),
\end{gathered}
$$

which, for $s \in \mathbb{N} \backslash\{1\}$, is recorded (among other places) in Prudnikov et al. [18, p. 388, Entry (2.5.4.1)] (see also Gradshteyn and Ryzhik [12, p. 418, Entry 3.748(3)]).

The integral formula (2.4) also simplifies when $\omega=3$ and $\omega=6$, giving us the following special cases:

$$
\begin{gathered}
\int_{0}^{\pi / 3} t^{s} \csc ^{2} t d t=-\frac{1}{\sqrt{3}}\left(\frac{\pi}{3}\right)^{s}-2 s\left(\frac{\pi}{3}\right)^{s-1} \sum_{k=0}^{\infty} \frac{\zeta(2 k)}{(s+2 k-1) 3^{2 k}} \\
(\Re(s)>1)
\end{gathered}
$$

and

$$
\begin{gathered}
\int_{0}^{\pi / 6} t^{s} \csc ^{2} t d t=-\sqrt{3}\left(\frac{\pi}{6}\right)^{s-1}-2 s\left(\frac{\pi}{6}\right)^{s-1} \sum_{k=0}^{\infty} \frac{\zeta(2 k)}{(s+2 k-1) 6^{2 k}} \\
(\Re(s)>1),
\end{gathered}
$$

which do not seem to have been recorded earlier.

Next, in terms of the incomplete Gamma function $\gamma(z, \alpha)$ defined by

$$
\gamma(z, \alpha):=\int_{0}^{\alpha} t^{z-1} e^{-t} d t
$$




$$
(\mathfrak{R}(z)>0 ; \quad|\arg (\alpha)| \leqq \pi-\varepsilon ; \quad 0<\varepsilon<\pi),
$$

it is easily seen that

$$
\int_{0}^{\tau} t^{\lambda-1} e^{-\mu t} d t=\mu^{-\lambda} \gamma(\lambda, \mu \tau) \quad(\Re(\lambda)>0)
$$

Thus, by writing

$$
\cot t=i\left(1+\frac{2}{e^{2 i t}-1}\right) \quad(i:=\sqrt{-1})
$$

and making use of (2.10), it is not difficult to deduce from (2.2) that

$$
\begin{gathered}
\mathcal{I}_{s}(\omega)=-\left(\frac{\pi}{\omega}\right)^{s}\left[\cot \left(\frac{\pi}{\omega}\right)+i\right]-2 i s \sum_{k=1}^{\infty} \frac{\gamma(s,-2 k \pi i / \omega)}{(-2 k i)^{s}} \\
(\mathfrak{R}(s)>1 ; \quad \omega>1) .
\end{gathered}
$$

Setting $s=2 n(n \in \mathbb{N})$ and noting that (cf., e.g., [12, p. 940, Entry 8.532(1)]

$$
\gamma(m+1, \alpha)=m !\left(1-e^{-\alpha} \sum_{j=0}^{m} \frac{\alpha^{j}}{j !}\right) \quad\left(m \in \mathbb{N}_{0}:=\mathbb{N} \cup\{0\}\right),
$$

(2.12) yields the integral formula:

$$
\begin{aligned}
& \mathcal{I}_{2 n}(\omega)=-\left(\frac{\pi}{\omega}\right)^{2 n} \cot \left(\frac{\pi}{\omega}\right)-(2 n) !\left(\frac{\pi}{\omega}\right)^{2 n-1}\left[\frac{1}{(2 n-1) !} \sum_{k=1}^{\infty} \frac{\cos (2 k \pi / \omega)}{k}\right. \\
&+\sum_{j=1}^{n-1} \frac{(-1)^{j}}{(2 n-2 j-1) !}\left(\frac{\omega}{2 \pi}\right)^{2 j} \sum_{k=1}^{\infty} \frac{\cos (2 k \pi / \omega)}{k^{2 j+1}} \\
&\left.+\sum_{j=1}^{n} \frac{(-1)^{j}}{(2 n-2 j) !}\left(\frac{\omega}{2 \pi}\right)^{2 j-1} \sum_{k=1}^{\infty} \frac{\sin (2 k \pi / \omega)}{k^{2 j}}\right] \\
&(n \in \mathbb{N} ; \omega>1),
\end{aligned}
$$

it being assumed here, and throughout this paper, that an empty sum is (as usual) nil. On the other hand, if we set $s=2 n+1(n \in \mathbb{N})$ in (2.12) and apply the reduction formula (2.13), we shall obtain

$$
\begin{aligned}
\mathcal{I}_{2 n+1}(\omega)= & -\left(\frac{\pi}{\omega}\right)^{2 n+1} \cot \left(\frac{\pi}{\omega}\right)+(-1)^{n} \frac{(2 n+1) !}{2^{2 n}} \zeta(2 n+1) \\
& -(2 n+1) !\left(\frac{\pi}{\omega}\right)^{2 n}\left[\frac{1}{(2 n) !} \sum_{k=1}^{\infty} \frac{\cos (2 k \pi / \omega))}{k}+\sum_{j=1}^{n} \frac{(-1)^{j}}{(2 n-2 j) !}\left(\frac{\omega}{2 \pi}\right)^{2 j}\right. \\
& \left.\cdot \sum_{k=1}^{\infty} \frac{\cos (2 k \pi / \omega)}{k^{2 j+1}}+\sum_{j=1}^{n} \frac{(-1)^{j}}{(2 n-2 j+1) !}\left(\frac{\omega}{2 \pi}\right)^{2 j-1} \sum_{k=1}^{\infty} \frac{\sin (2 k \pi / \omega)}{k^{2 j}}\right] \\
& (n \in \mathbb{N} ; \quad \omega>1) .
\end{aligned}
$$


Since (cf., e.g., [13, p. 239, Entry 17.2.5)] with $y=0$ )

$$
\sum_{k=1}^{\infty} \frac{\cos (k x)}{k}=-\log \left[2 \sin \left(\frac{1}{2} x\right)\right] \quad(0<x<2 \pi)
$$

in terms of the generalized Clausen functions $\mathrm{C} \ell_{2 n}$ and $\mathrm{C} \ell_{2 n+1}$ defined by (cf. Lewin [16, p. 191, Equations (7.9) and (7.10)])

$$
\mathrm{C} \ell_{2 n}(x):=\sum_{k=1}^{\infty} \frac{\sin (k x)}{k^{2 n}} \quad \text { and } \quad C \ell_{2 n+1}(x):=\sum_{k=1}^{\infty} \frac{\cos (k x)}{k^{2 n+1}} \quad(n \in \mathbb{N}),
$$

it is easily seen from our evaluations (2.4), (2.14), and (2.15) that

$$
\begin{aligned}
& \sum_{k=0}^{\infty} \frac{\zeta(2 k)}{(2 k+2 n-1) \omega^{2 k}}=-\frac{1}{2} \log \left[2 \sin \left(\frac{\pi}{\omega}\right)\right] \\
&+\frac{(2 n-1) !}{2}\left[\sum_{j=1}^{n-1} \frac{(-1)^{j}}{(2 n-2 j-1) !}\left(\frac{\omega}{2 \pi}\right)^{2 j} \mathrm{C} \ell_{2 j+1}\left(\frac{2 \pi}{\omega}\right)\right. \\
&+\left.\sum_{j=1}^{n} \frac{(-1)^{j}}{(2 n-2 j) !}\left(\frac{\omega}{2 \pi}\right)^{2 j-1} \mathrm{C} \ell_{2 j}\left(\frac{2 \pi}{\omega}\right)\right] \\
&(n \in \mathbb{N} ; \quad \omega>1)
\end{aligned}
$$

and

$$
\begin{aligned}
\sum_{k=0}^{\infty} \frac{\zeta(2 k)}{(2 k+2 n) \omega^{2 k}} & =(-1)^{n-1} \frac{(2 n) !}{2^{2 n+1}}\left(\frac{\omega}{\pi}\right)^{2 n} \zeta(2 n+1)-\frac{1}{2} \log \left[2 \sin \left(\frac{\pi}{\omega}\right)\right] \\
& +\frac{(2 n) !}{2} \sum_{j=1}^{n} \frac{(-1)^{j}}{(2 n-2 j+1) !}\left(\frac{\omega}{2 \pi}\right)^{2 j}\left[(2 n-2 j+1) \mathrm{C} \ell_{2 j+1}\left(\frac{2 \pi}{\omega}\right)+\frac{2 \pi}{\omega} \mathrm{C} \ell_{2 j}\left(\frac{2 \pi}{\omega}\right)\right]
\end{aligned}
$$

$$
(n \in \mathbb{N} ; \quad \omega>1)
$$

In its special case when $n=1,(2.18)$ would readily yield a known result [13, p. 356, Entry (54.5.4)] in the form:

$$
\sum_{k=0}^{\infty} \frac{\zeta(2 k))}{(2 k+1) \omega^{2 k}}=-\frac{1}{2} \log \left[2 \sin \left(\frac{\pi}{\omega}\right)\right]-\frac{\omega}{4 \pi} \mathrm{C} \ell_{2}\left(\frac{2 \pi}{\omega}\right) \quad(\omega>1) .
$$

Moreover, since [16, pp. 103-104, Equations (4.14) and (4.15)]

$$
\mathrm{C} \ell_{2}\left(\frac{1}{2} \pi\right)=G=-\mathrm{C} \ell_{2}\left(\frac{3}{2} \pi\right)
$$


where $G$ denotes Catalan's constant defined by

$$
\begin{aligned}
G & :=\sum_{k=0}^{\infty} \frac{(-1)^{k}}{(2 k+1)^{2}}=-\int_{0}^{\pi / 2} \log \left[2 \sin \left(\frac{1}{2} t\right)\right] d t \\
& \cong 0.915965594177219015 \ldots,
\end{aligned}
$$

by setting $\omega=4$ and $\omega=\frac{4}{3}$ in $(2.20)$, we obtain its further special cases:

$$
\sum_{k=0}^{\infty} \frac{\zeta(2 k)}{(2 k+1) 4^{2 k}}=-\frac{G}{\pi}-\frac{1}{4} \log 2
$$

and

$$
\sum_{k=0}^{\infty} \frac{\zeta(2 k)}{2 k+1}\left(\frac{3}{4}\right)^{2 k}=\frac{G}{3 \pi}-\frac{1}{4} \log 2
$$

in terms of the Catalan constant $G$ defined by (2.22).

\section{Series Representations for $\zeta(2 n+1)$}

In every situation in which the Clausen functions $\mathrm{C} \ell_{2 n}(x)$ and $\mathrm{C} \ell_{2 n+1}(x)$ can be expressed in closed forms, each of our formulas (2.18) and (2.19) will readily yield a series representation for $\zeta(2 n+1)(n \in \mathbb{N})$. We begin by considering the following rather simple special case.

Case 1. Let $\omega=2$. It immediately follows from the definitions (1.1) and (2.17) that

$$
\mathrm{C} \ell_{2 n}(\pi)=0 \quad \text { and } \quad \mathrm{C}_{2 n+1}(\pi)=\left(2^{-2 n}-1\right) \zeta(2 n+1) \quad(n \in \mathbb{N}) .
$$

Thus the formulas (2.18) and (2.19) yield the series representations:

$$
\begin{aligned}
\zeta(2 n+1)=(-1)^{n-1} \frac{(2 \pi)^{2 n}}{(2 n+1) !\left(2^{2 n}-1\right)}\left[\log 2+2 \sum_{k=0}^{\infty} \frac{\zeta(2 k)}{(2 k+2 n+1) 2^{2 k}}\right. \\
\left.+(2 n+1) ! \sum_{j=1}^{n-1} \frac{(-1)^{j}}{(2 n-2 j+1) !}\left(\frac{2^{2 j}-1}{(2 \pi)^{2 j}}\right) \zeta(2 j+1)\right] \quad(n \in \mathbb{N})
\end{aligned}
$$

and

$$
\begin{aligned}
\zeta(2 n+1)=(-1)^{n-1} \frac{(2 \pi)^{2 n}}{(2 n) !\left(2^{2 n+1}-1\right)} & {\left[\log 2+\sum_{k=0}^{\infty} \frac{\zeta 2 k)}{(k+n) 2^{2 k}}\right.} \\
& \left.+(2 n) ! \sum_{j=1}^{n-1} \frac{(-1)^{j}}{(2 n-2 j) !}\left(\frac{2^{2 j}-1}{(2 \pi)^{2 j}}\right) \zeta(2 j+1)\right] \quad(n \in \mathbb{N}),
\end{aligned}
$$

respectively.

For $n=1,(3.2)$ immediately reduces to the following series representation for $\zeta(3)$ :

$$
\zeta(3)=\frac{2 \pi^{2}}{9}\left(\log 2+2 \sum_{k=0}^{\infty} \frac{\zeta(2 k)}{(2 k+3) 2^{2 k}}\right),
$$


which was proven independently by (among others) Glasser [10, p. 446, Equation (12)], Zhang and Williams [24, p. 1585, Equation (2.13)], and Dąbrowski [7, p. 206] (see also Chen and Srivastava [5, p. 183, Equation (2.15)]). And a special case of (3.3) when $n=1$ yields (cf. Dąbrowski [7, p. 202]; see also Chen and Srivastava [5, p. 191, Equation (3.19)])

$$
\zeta(3)=\frac{2 \pi^{2}}{7}\left(\log 2+\sum_{k=0}^{\infty} \frac{\zeta(2 k)}{(k+1) 2^{2 k}}\right) .
$$

In view of the known sum:

$$
\sum_{k=0}^{\infty} \frac{\zeta(2 k)}{(2 k+1) 2^{2 k}}=-\frac{1}{2} \log 2,
$$

which incidentally results also from (2.18) in the special case when $\omega=2$ and $n=1$, Euler's formula (1.5) is indeed a simple consequence of (3.5).

We remark in passing that an integral representation for $\zeta(2 n+1)$, which is easily seen to be equivalent to the series representation (3.3), was given by Dąbrowski [7, p. 203, Equation (16)], who [7, p. 206] mentioned the existence of (but did not fully state) the series representation (3.2) as well. The series representation (3.3) is derived also in a forthcoming paper by Borwein et al. (cf. [4, Equation (57)]).

The generalized Clausen function $\mathrm{C}_{2 n+1}(x)$ defined in (2.17) is known to be expressible in a closed form in at least three other cases, and we have (cf. Lewin [16, p. 198])

$$
\begin{gathered}
\mathrm{C} \ell_{2 n+1}\left(\frac{1}{2} \pi\right)=-2^{-2 n-1}\left(1-2^{-2 n}\right) \zeta(2 n+1) \quad(n \in \mathbb{N}), \\
\mathrm{C} \ell_{2 n+1}\left(\frac{1}{3} \pi\right)=\frac{1}{2}\left(1-2^{-2 n}\right)\left(1-3^{-2 n}\right) \zeta(2 n+1) \quad(n \in \mathbb{N}),
\end{gathered}
$$

and

$$
\mathrm{C} \ell_{2 n+1}\left(\frac{2}{3} \pi\right)=-\frac{1}{2}\left(1-3^{-2 n}\right) \zeta(2 n+1) \quad(n \in \mathbb{N}) .
$$

With a view to evaluating the generalized Clausen function $\mathrm{C}_{2 n}(x)$, also defined in (2.17), when

$$
x=\frac{1}{2} \pi, \quad \frac{1}{3} \pi, \quad \text { and } \quad \frac{2}{3} \pi,
$$

we recall the following result recorded (for example) by Hansen [13, p. 223, Entry (14.4.3)]:

$$
\begin{aligned}
& \sum_{k=1}^{\infty} \frac{\sin (k x+y)}{k^{s}}=\frac{(2 \pi)^{s}}{2 \Gamma(s)} \csc (\pi s)\left[\cos \left(y-\frac{1}{2} \pi s\right) \zeta\left(1-s, \frac{x}{2 \pi}\right)\right. \\
&\left.-\cos \left(y+\frac{1}{2} \pi s\right) \zeta\left(1-s, 1-\frac{x}{2 \pi}\right)\right] \\
&(\mathfrak{R}(s)>1 ; \quad 0<x<2 \pi),
\end{aligned}
$$

which, for

$$
x=\frac{2 \pi}{\omega} \quad(\omega>1) \quad \text { and } \quad y=0
$$


reduces at once to the form:

$$
\begin{gathered}
\sum_{k=1}^{\infty} \frac{\sin (2 k \pi / \omega)}{k^{s}}=\frac{(2 \pi)^{s}}{4 \Gamma(s)} \csc \left(\frac{1}{2} \pi s\right)\left[\zeta\left(1-s, \frac{1}{\omega}\right)-\zeta\left(1-s, 1-\frac{1}{\omega}\right)\right] \\
(\Re(s)>1 ; \quad \omega>1) .
\end{gathered}
$$

Now, making use of the identity:

$$
\zeta(s)=\frac{1}{q^{s}-1} \sum_{j=1}^{q-1} \zeta\left(s, \frac{j}{q}\right) \quad(q \in \mathbb{N} \backslash\{1\}),
$$

which follows readily from the definitions (1.1) and (1.2), the Rademacher formula (cf., e.g., Magnus et al. $[17$, p. 23]):

$$
\begin{aligned}
\zeta\left(s, \frac{p}{q}\right)=2 \Gamma(1-s)(2 q \pi)^{s-1} & {\left[\sin \left(\frac{1}{2} \pi s\right) \sum_{j=1}^{q} \cos \left(\frac{2 p j \pi}{q}\right) \zeta\left(1-s, \frac{j}{q}\right)\right.} \\
& \left.+\cos \left(\frac{1}{2} \pi s\right) \sum_{j=1}^{q} \sin \left(\frac{2 p j \pi}{q}\right) \zeta\left(1-s, \frac{j}{q}\right)\right] \quad(p, q \in \mathbb{N}),
\end{aligned}
$$

as well as the familiar special case of (3.13) when $p=q=1$ :

$$
\zeta(s)=2(2 \pi)^{s-1} \sin \left(\frac{1}{2} \pi s\right) \Gamma(1-s) \zeta(1-s)
$$

or, equivalently,

$$
\zeta(1-s)=2(2 \pi)^{-s} \cos \left(\frac{1}{2} \pi s\right) \Gamma(s) \zeta(s),
$$

we find from (3.11) and the relevant definition in (2.17) that

$$
\begin{gathered}
\mathrm{C} \ell_{2 n}\left(\frac{1}{2} \pi\right)=2^{1-4 n} \zeta\left(2 n, \frac{1}{4}\right)-\left(1-2^{-2 n}\right) \zeta(2 n) \quad(n \in \mathbb{N}), \\
\mathrm{C} \ell_{2 n}\left(\frac{1}{3} \pi\right)=\sqrt{3}\left[6^{-2 n}\left\{\zeta\left(2 n, \frac{1}{3}\right)+\zeta\left(2 n, \frac{1}{6}\right)\right\}-\frac{1-3^{-2 n}}{2} \zeta(2 n)\right] \quad(n \in \mathbb{N}),
\end{gathered}
$$

and

$$
\mathrm{C} \ell_{2 n}\left(\frac{2}{3} \pi\right)=\sqrt{3}\left[3^{-2 n} \zeta\left(2 n, \frac{1}{3}\right)-\frac{1-3^{-2 n}}{2} \zeta(2 n)\right] \quad(n \in \mathbb{N}),
$$

in which each $\zeta(2 n)$ can be replaced by its value in terms of the Bernoulli numbers $B_{2 n}$ by appealing to the well-known relationship (cf., e.g., [17, p. 19]):

$$
\zeta(2 n)=(-1)^{n+1} \frac{(2 \pi)^{2 n}}{2(2 n) !} B_{2 n} \quad\left(n \in \mathbb{N}_{0}\right) .
$$


Much more general trigonometric sums than the ones involved in (3.7) to (3.9) and (3.16) to (3.18) can also be evaluated by applying the aforementioned technique or (alternatively) by appealing appropriately to the elementary series identity:

$$
\sum_{k=1}^{\infty} f(k)=\sum_{j=1}^{q} \sum_{k=0}^{\infty} f(q k+j) \quad(q \in \mathbb{N})
$$

and its straightforward consequence (3.12). Thus, for example, we can derive a generalization of (3.7) in the form:

$$
\sum_{k=1}^{\infty} \frac{\cos \left(\frac{1}{2} k \pi\right)}{k^{s}}=-2^{-s}\left(1-2^{1-s}\right) \zeta(s) \quad(\Re(s)>1)
$$

which immediately yields (3.7) in the special case when $s=2 n+1(n \in \mathbb{N})$. Analogous generalizations of the remaining evaluations of the Clausen functions $\mathrm{C} \ell_{2 n+1}(x)$ and $\mathrm{C} \ell_{2 n}(x)$ can be derived similarly; we choose to skip the details involved.

In view of the evaluations (3.7) to (3.9) and (3.16) to (3.18), we are led easily to the following additional special cases of our general results (2.18) and (2.19).

Case 2. Let $\omega=3$. Then, making use of (3.9) and (3.18) in each of our formulas (2.18) and (2.19), we obtain the series representations:

$$
\begin{aligned}
\zeta(2 n+1) & =(-1)^{n-1} \frac{(2 \pi)^{2 n}}{(2 n+1) !\left(3^{2 n}-1\right)}\left[\log 3+4 \sum_{k=0}^{\infty} \frac{\zeta(2 k)}{(2 k+2 n+1) 3^{2 k}}\right. \\
& +(2 n+1) ! \sum_{j=1}^{n-1} \frac{(-1)^{j}}{(2 n-2 j+1) !}\left(\frac{3^{2 j}-1}{(2 \pi)^{2 j}}\right) \zeta(2 j+1) \\
& \left.-\frac{(2 n+1) !}{\sqrt{3}} \sum_{j=1}^{n+1} \frac{(-1)^{j}}{(2 n-2 j+2) !} \frac{2 \zeta\left(2 j, \frac{1}{3}\right)-\left(3^{2 j}-1\right) \zeta(2 j)}{(2 \pi)^{2 j-1}}\right] \quad(n \in \mathbb{N})
\end{aligned}
$$

and

$$
\begin{aligned}
\zeta(2 n+1) & =(-1)^{n-1} \frac{(2 \pi)^{2 n}}{(2 n) !\left(3^{2 n+1}-1\right)}\left[\log 3+2 \sum_{k=0}^{\infty} \frac{\zeta(2 k)}{(k+n) 3^{2 k}}\right. \\
& +(2 n) ! \sum_{j=1}^{n-1} \frac{(-1)^{j}}{(2 n-2 j) !}\left(\frac{3^{2 j}-1}{(2 \pi)^{2 j}}\right) \zeta(2 j+1) \\
& \left.-\frac{(2 n) !}{\sqrt{3}} \sum_{j=1}^{n} \frac{(-1)^{j}}{(2 n-2 j+1) !} \frac{2 \zeta\left(2 j, \frac{1}{3}\right)-\left(3^{2 j}-1\right) \zeta(2 j)}{(2 \pi)^{2 j-1}}\right] \quad(n \in \mathbb{N}) .
\end{aligned}
$$


In particular, when $n=1,(3.22)$ and (3.23) yield the following (presumably new) series representations for $\zeta(3)$ :

$$
\begin{aligned}
\zeta(3) & =\frac{\pi^{2}}{12}\left[\log 3+4 \sum_{k=0}^{\infty} \frac{\zeta(2 k)}{(2 k+3) 3^{2 k}}\right. \\
& +2 \sqrt{3} \sum_{j=1}^{2} \frac{(-1)^{j-1}}{(4-2 j) !} \frac{2 \zeta\left(2 j, \frac{1}{3}\right)-\left(3^{2 j}-1\right) \zeta(2 j)}{(2 \pi)^{2 j-1}}
\end{aligned}
$$

and

$$
\zeta(3)=\frac{\pi^{2}}{13}\left[\log 3+2 \sum_{k=0}^{\infty} \frac{\zeta(2 k)}{(k+1) 3^{2 k}}+\frac{2}{\pi \sqrt{3}}\left\{\zeta\left(2, \frac{1}{3}\right)-4 \zeta(2)\right\}\right] .
$$

Case 3. Let $\omega=4$. Then, by applying the evaluations (3.7) and (3.16) in each of our formulas (2.18) and (2.19), we obtain the series representations:

$$
\begin{aligned}
\zeta(2 n+1) & =(-1)^{n-1} \frac{(2 \pi)^{2 n}}{(2 n+1) !\left(2^{2 n}-1\right)}\left[\log 2+4 \sum_{k=0}^{\infty} \frac{\zeta(2 k)}{(2 k+2 n+1) 4^{2 k}}\right. \\
& +(2 n+1) ! \sum_{j=1}^{n-1} \frac{(-1)^{j}}{(2 n-2 j+1) !}\left(\frac{2^{2 j}-1}{(2 \pi)^{2 j}}\right) \zeta(2 j+1) \\
& \left.-(2 n+1) ! \sum_{j=1}^{n+1} \frac{(-1)^{j}}{(2 n-2 j+2) !} \frac{\zeta\left(2 j, \frac{1}{4}\right)-2^{2 j-1}\left(2^{2 j}-1\right) \zeta(2 j)}{(2 \pi)^{2 j-1}}\right] \quad(n \in \mathbb{N})
\end{aligned}
$$

and

$$
\begin{aligned}
\zeta(2 n+1) & =(-1)^{n-1} \frac{(2 \pi)^{2 n}}{(2 n) !\left(2^{4 n+1}+2^{2 n}-1\right)}\left[\log 2+2 \sum_{k=0}^{\infty} \frac{\zeta(2 k)}{(k+n) 4^{2 k}}\right. \\
& +(2 n) ! \sum_{j=1}^{n-1} \frac{(-1)^{j}}{(2 n-2 j) !}\left(\frac{2^{2 j}-1}{(2 \pi)^{2 j}}\right) \zeta(2 j+1) \\
& \left.-(2 n) ! \sum_{j=1}^{n} \frac{(-1)^{j}}{(2 n-2 j+1) !} \frac{\zeta\left(2 j, \frac{1}{4}\right)-2^{2 j-1}\left(2^{2 j}-1\right) \zeta(2 j)}{(2 \pi)^{2 j-1}}\right] \quad(n \in \mathbb{N}) .
\end{aligned}
$$

In their special cases when $n=1,(3.26)$ and (3.27) yield the following (presumably new) series representations for $\zeta(3)$ :

$$
\begin{aligned}
\zeta(3) & =\frac{2 \pi^{2}}{9}\left[\log 2+4 \sum_{k=0}^{\infty} \frac{\zeta 2 k)}{(2 k+3) 4^{2 k}}\right. \\
& \left.+6 \sum_{j=1}^{2} \frac{(-1)^{j-1}}{(4-2 j) !} \frac{\zeta\left(2 j, \frac{1}{4}\right)-2^{2 j-1}\left(2^{2 j}-1\right) \zeta(2 j)}{(2 \pi)^{2 j-1}}\right]
\end{aligned}
$$


and

$$
\zeta(3)=\frac{2 \pi^{2}}{35}\left[\log 2+2 \sum_{k=0}^{\infty} \frac{\zeta(2 k)}{(k+1) 4^{2 k}}+\frac{1}{\pi}\left\{\zeta\left(2, \frac{1}{4}\right)-6 \zeta(2)\right\}\right] .
$$

Case 4. Let $\omega=6$. Then, in view of the evaluations (3.8) and (3.17), our formulas (2.18) and (2.19) give us the series representations:

$$
\begin{aligned}
\zeta(2 n+1) & =(-1)^{n-1} \frac{(2 \pi)^{2 n}}{\left(2^{2 n}-1\right)\left(3^{2 n}-1\right)}\left[-\frac{4}{(2 n+1) !} \sum_{k=0}^{\infty} \frac{\zeta(2 k)}{(2 k+2 n+1) 6^{2 k}}\right. \\
& +\sum_{j=1}^{n-1} \frac{(-1)^{j}}{(2 n-2 j+1) !}\left(\frac{\left(2^{2 j}-1\right)\left(3^{2 j}-1\right)}{(2 \pi)^{2 j}}\right) \zeta(2 j+1) \\
& \left.+\frac{1}{\sqrt{3}} \sum_{j=1}^{n+1} \frac{(-1)^{j}}{(2 n-2 j+2) !} \frac{\zeta\left(2 j, \frac{1}{3}\right)+\zeta\left(2 j, \frac{1}{6}\right)-2^{2 j-1}\left(3^{2 j}-1\right) \zeta(2 j)}{(2 \pi)^{2 j-1}}\right]
\end{aligned}
$$$$
(n \in \mathbb{N})
$$

and

$$
\begin{aligned}
\zeta(2 n+1) & =(-1)^{n-1} \frac{(2 \pi)^{2 n}}{2^{2 n}+3^{2 n}+6^{2 n}-1}\left[\frac{2}{(2 n) !} \sum_{k=0}^{\infty} \frac{\zeta(2 k)}{(k+n) 6^{2 k}}\right. \\
& -\sum_{j=1}^{n-1} \frac{(-1)^{j}}{(2 n-2 j) !}\left(\frac{\left(2^{2 j}-1\right)\left(3^{2 j}-1\right)}{(2 \pi)^{2 j}}\right) \zeta(2 j+1) \\
& \left.-\frac{1}{\sqrt{3}} \sum_{j=1}^{n} \frac{(-1)^{j}}{(2 n-2 j+1) !} \frac{\zeta\left(2 j, \frac{1}{3}\right)+\zeta\left(2 j, \frac{1}{6}\right)-2^{2 j-1}\left(3^{2 j}-1\right) \zeta(2 j)}{(2 \pi)^{2 j-1}}\right]
\end{aligned}
$$

$(n \in \mathbb{N})$

For $n=1$, these last results (3.30) and (3.31) yield the following (presumably new) series representations for $\zeta(3)$ :

$$
\begin{aligned}
\zeta(3) & =-\frac{\pi^{2}}{18}\left[2 \sum_{k=0}^{\infty} \frac{\zeta(2 k)}{(2 k+3) 6^{2 k}}\right. \\
& \left.+\sqrt{3} \sum_{j=1}^{2} \frac{(-1)^{j-1}}{(4-2 j) !} \frac{\zeta\left(2 j, \frac{1}{3}\right)+\left(2 j, \frac{1}{6}\right)-2^{2 j-1}\left(3^{2 j}-1\right) \zeta(2 j)}{(2 \pi)^{2 j-1}}\right]
\end{aligned}
$$

and

$$
\zeta(3)=\frac{\pi^{2}}{12}\left[\sum_{k=0}^{\infty} \frac{\zeta(2 k)}{(k+1) 6^{2 k}}+\frac{1}{2 \pi \sqrt{3}}\left\{\zeta\left(2, \frac{1}{3}\right)+\zeta\left(2, \frac{1}{6}\right)-16 \zeta(2)\right\}\right] .
$$




\section{An Alternative Derivation of the Series Representation (3.2)}

In view of the following known representation of $\zeta(s)$ as a Mellin transform [17, p. 21]:

$$
\zeta(s)=\frac{2^{s-1}}{\left(1-2^{1-s}\right) \Gamma(s+1)} \int_{0}^{\infty} t^{s} \operatorname{sech}^{2} t d t \quad(\Re(s)>-1 ; s \neq 1),
$$

we propose to give here an interesting alternative derivation of the very first of the set of eight series representations for $\zeta(2 n+1)(n \in \mathbb{N})$, which we have presented in the preceding section. Indeed, from the work of Glasser [10, p. 445, Equation (4) with $\alpha \rightarrow 0$ ], it is known also that

$$
\begin{aligned}
I & :=\int_{0}^{\infty} \frac{\left(\tan ^{-1} z\right)^{2 n}}{z^{2}} d z=\int_{0}^{\pi / 2} t^{2 n} \csc ^{2} t d t \\
& =(-1)^{n} 2^{1-2 n} \mathfrak{I}\left\{\int_{0}^{1} \frac{(\log u-i \pi)^{2 n}}{(u+1)^{2}} d u\right\} \quad(n \in \mathbb{N}) .
\end{aligned}
$$

Now evaluate the cosecant integral in (4.2) by means of the known result (2.5) with $s=2 n$ $(n \in \mathbb{N})$. Thus, setting $\log u=-2 t$, we find from the last member of (4.2) that

$$
\sum_{k=0}^{\infty} \frac{\zeta(2 k)}{(2 k+2 n-1) 2^{2 k}}=\frac{(-1)^{n-1}}{4 n}\left(\frac{2}{\pi}\right)^{2 n-1} \mathfrak{I}\left\{\int_{0}^{\infty}\left(t+\frac{1}{2} i \pi\right)^{2 n} \operatorname{sech}^{2} t d t\right\} \quad(n \in \mathbb{N}) .
$$

By the binomial expansion, we have

$$
\begin{aligned}
\left(t+\frac{1}{2} i \pi\right)^{2 n} & =\sum_{k=0}^{2 n}\left(\begin{array}{c}
2 n \\
k
\end{array}\right) t^{k}\left(\frac{1}{2} i \pi\right)^{2 n-k} \\
& =\sum_{j=0}^{n}(-1)^{n-j}\left(\begin{array}{c}
2 n \\
2 j
\end{array}\right) t^{2 j}\left(\frac{\pi}{2}\right)^{2 n-2 j}-i \sum_{j=0}^{n-1}(-1)^{n-j}\left(\begin{array}{c}
2 n \\
2 j+1
\end{array}\right) t^{2 j+1}\left(\frac{\pi}{2}\right)^{2 n-2 j-1} \\
& \left(n \in \mathbb{N}_{0}\right),
\end{aligned}
$$

where, as also elsewhere in this work, an empty sum in interpreted to be nil. Upon replacing $n$ in (4.3) by $n+1$, and making use of (4.4), we obtain

$$
\begin{aligned}
\sum_{k=0}^{\infty} \frac{\zeta(2 k)}{(2 k+2 n+1) 2^{2 k}}=\frac{1}{2} \sum_{j=0}^{n} \frac{(-1)^{j-1}}{2 j+1}\left(\begin{array}{c}
2 n+1 \\
2 j
\end{array}\right)\left(\frac{2}{\pi}\right)^{2 j} \\
\cdot \int_{0}^{\infty} t^{2 j+1} \operatorname{sech}^{2} t d t \quad\left(n \in \mathbb{N}_{0}\right) .
\end{aligned}
$$

The infinite integral in (4.5) can be evaluated by means of the known result (4.1) except when $j=0$, in which case it is easily seen by integrating by parts that (cf. [12, p. 353, Entry 3.527(4)])

$$
\int_{0}^{\infty} t \operatorname{sech}^{2} t d t=\lim _{t \rightarrow \infty}(t \tanh t-\log \cosh t)=\log 2 .
$$

We thus find from (4.5) that

$$
\sum_{k=0}^{\infty} \frac{\zeta(2 k)}{(2 k+2 n+1) 2^{2 k}}=\frac{1}{2} \sum_{j=1}^{n}(-1)^{j-1}\left(\begin{array}{c}
2 n+1 \\
2 j
\end{array}\right) \frac{(2 j) !\left(2^{2 j}-1\right)}{(2 \pi)^{2 j}} \zeta(2 j+1)-\frac{1}{2} \log 2 \quad\left(n \in \mathbb{N}_{0}\right) .
$$


For $n=0$, (4.7) immediately yields the known sum (3.6), which (as we observed in the proceding section) is derivable also from (2.18) for $\omega=2$ and $n=1$. More interestingly, just as we indicated in Section 3, since

$$
\frac{1}{(2 k+1)(2 k+2)}=\frac{1}{2 k+1}-\frac{1}{2 k+2},
$$

by suitably combining the series representation (3.5) with (3.6), we can easily deduce Euler's formula (1.5). In the case when $n \in \mathbb{N}$, by separating the term for $j=n$ in (4.7), we readily obtain the desired series representation (3.2) in its (obviously equivalent) form:

$$
\begin{aligned}
\zeta(2 n+1) & =(-1)^{n-1} \frac{(2 \pi)^{2 n}}{(2 n+1) !\left(2^{2 n}-1\right)}\left[\log 2+2 \sum_{k=0}^{\infty} \frac{\zeta(2 k)}{(2 k+2 n+1) 2^{2 k}}\right. \\
& \left.+\sum_{j=1}^{n-1}(-1)^{j-1}\left(\begin{array}{c}
2 n+1 \\
2 j
\end{array}\right) \frac{(2 j) !\left(2^{2 j}-1\right)}{(2 \pi)^{2 j}} \zeta(2 j+1)\right] \quad(n \in \mathbb{N}) .
\end{aligned}
$$

\section{A Unification of the Series Evaluations (2.18) and (2.19)}

With a view to unifying the series evaluations (2.18) and (2.19), we begin by considering the formulas (2.2) and (2.4), which readily yield the following representation:

$$
\begin{gathered}
\mathcal{S}_{p}:=\sum_{k=0}^{\infty} \frac{\zeta(2 k)}{(2 k+p) \omega^{2 k}}=-\frac{\pi}{2 \omega} \int_{0}^{1} t^{p} \cot \left(\frac{\pi t}{\omega}\right) d t \\
(p \in \mathbb{N} ; \quad|\omega|>1),
\end{gathered}
$$

where, for the purpose of this section, we have only considered a special case when $s=p+1(p \in \mathbb{N})$.

Now change the variable of integration in (5.1) by letting

$$
t=-\frac{i \omega}{2 \pi} \log z .
$$

Since

$$
\int_{1}^{z} \frac{(\log t)^{p}}{t} d t=\frac{z^{p+1}}{p+1} \quad(p \in \mathbb{N})
$$

we thus find from (5.1) that

$$
\begin{gathered}
\mathcal{S}_{p}=\frac{\pi i}{2(p+1) \omega}+\frac{1}{2}\left(-\frac{i \omega}{2 \pi}\right)^{p} \int_{1}^{\Omega} \frac{(\log z)^{p}}{1-z} d z \\
(p \in \mathbb{N} ; \quad \omega \mid>1),
\end{gathered}
$$

where, for convenience,

$$
\Omega:=\exp \left(\frac{2 \pi i}{\omega}\right) \quad(|\omega|>1) .
$$

Finally, by setting

$$
z=1-(1-\Omega) t
$$


in (5.3), we obtain

$$
\mathcal{S}_{p}=\frac{\pi i}{2(p+1) \omega}-\frac{1}{2}\left(-\frac{i \omega}{2 \pi}\right)^{p} \int_{0}^{1}\{\log (1-(1-\Omega) t)\}^{p} \frac{d t}{t}
$$

or, equivalently,

$$
\sum_{k=0}^{\infty} \frac{\zeta(2 k)}{(2 k+p) \omega^{2 k}}=\frac{\pi i}{2(p+1) \omega}-\frac{p !}{2}\left(\frac{i \omega}{2 \pi}\right)^{p} S_{1, p}\left(1-e^{2 \pi i / \omega}\right)
$$

in terms of Nielsen's generalized Polylogarithmic function $S_{n, p}(z)$ defined by $(c f .$, e.g., Kölbig [15, p. 1233, Equation (1.3)])

$$
\begin{gathered}
S_{n, p}(z):=\frac{(-1)^{n+p-1}}{(n-1) ! p !} \int_{0}^{1}(\log t)^{n-1}\{\log (1-z t)\}^{p} \frac{d t}{t} \\
(n, p \in \mathbb{N} ; \quad z \in \mathbb{C}),
\end{gathered}
$$

which is known to play a rôle in the computation of higher-order radiative corrections in quantum electrodynamics. For the ordinary Polylogarithmic function $\operatorname{Li}_{n}(z)$ defined by

$$
\begin{gathered}
\operatorname{Li}_{n}(z):=\frac{(-1)^{n-1}}{(n-2) !} \int_{0}^{1}(\log t)^{n-1} \log (1-z t) \frac{d t}{t}=S_{n-1,1}(z) \\
(n \in \mathbb{N} \backslash\{1\} ; \quad z \in \mathbb{C})
\end{gathered}
$$

or, more generally, by

$$
\begin{gathered}
\operatorname{Li}_{s}(z):=\sum_{k=1}^{\infty} \frac{z^{k}}{k^{s}} \\
(s \in \mathbb{C} \text { when }|z|<1 ; \quad \mathfrak{R}(s)>1 \text { when }|z|=1),
\end{gathered}
$$

it is known that (cf. [15, p. 1240, Equation (5.6)])

$$
S_{1, p}(z)=\zeta(p+1)+\sum_{k=0}^{p} \frac{(-1)^{k-1}}{k !}\{\log (1-z)\}^{k} \operatorname{Li}_{p-k+1}(1-z) .
$$

Thus, in view of (5.10), we have proved the following unification of our series evaluations (2.18) and (2.19):

$$
\begin{aligned}
& \sum_{k=0}^{\infty} \frac{\zeta(2 k)}{(2 k+p) \omega^{2 k}}=\frac{\pi i}{2(p+1) \omega}-\frac{1}{2} \log \left(1-e^{2 \pi i / \omega}\right) \\
&-\frac{p !}{2}\left(\frac{i \omega}{2 \pi}\right)^{p} \zeta(p+1)+\frac{1}{2} \sum_{k=1}^{p}\left(\begin{array}{l}
p \\
k
\end{array}\right) k !\left(\frac{i \omega}{2 \pi}\right)^{k} \operatorname{Li}_{k}\left(e^{2 \pi i / \omega}\right) \\
&(p \in \mathbb{N} ; \quad|\omega|>1)
\end{aligned}
$$


which, in view of (5.1), immediately yields the integral formula:

$$
\begin{aligned}
& \int_{0}^{1} t^{p} \cot (\nu t) d t=-\frac{i}{p+1}+\frac{1}{\nu} \log \left(1-e^{2 \nu i}\right) \\
&+ \frac{p !}{\nu}\left(\frac{i}{2 \nu}\right)^{p} \zeta(p+1)-\frac{1}{\nu} \sum_{k=1}^{p}\left(\begin{array}{l}
p \\
k
\end{array}\right) k !\left(\frac{i}{2 \nu}\right)^{k} \operatorname{Li}_{k+1}\left(e^{2 \nu i}\right) \\
&(p \in \mathbb{N} ; \quad \nu \in \mathbb{C} \backslash\{0\}) .
\end{aligned}
$$

There is yet another approach for the derivation of a closed-form expression for the infinite series in (5.11). This general (generating-function) approach is based upon a familiar integral representation for the Zeta function; it was fully described and illustrated by Adamchik and Srivastava [1], whose technique is also implemented in Mathematica (Version 3.0). Here we choose to present three examples which demonstrate how Mathematica (Version 4.0) for Linux can be used to evaluate the infinite series in (5.11) for certain values of the parameters $p$ and $\omega$.

$$
\begin{aligned}
& \operatorname{In}[1]:=\operatorname{Sum}[\text { Zeta }[2 k] /((2 k+1) 2\urcorner(2 k)),\{k, 1, \text { Infinity }\}] \\
& \text { Out }[1]=\frac{1-\log [2]}{2} \\
& \operatorname{In}[2]:=\operatorname{Sum}\left[\text { Zeta }[2 k] /\left((2 k+2) 2^{\urcorner}(2 k)\right),\{k, 1, \text { Infinity }\}\right] \\
& \operatorname{Out}[2]=\frac{\mathrm{Pi}^{2}-2 \mathrm{Pi}^{2} \log [2]+7 Z \operatorname{Zeta}[3]}{4 \mathrm{Pi}^{2}} \\
& \operatorname{In}[3]:=\operatorname{Sum}[\operatorname{Zeta}[2 k] /((2 k+3) 2\urcorner(2 k)),\{k, 1, \text { Infinity }\}] \\
& \text { Out }[3]=\frac{2 \mathrm{Pi}^{2}-6 \mathrm{Pi}^{2} \log [2]+27 Z \operatorname{Zeta}[3]}{12 \mathrm{Pi}^{2}}
\end{aligned}
$$

Since $\zeta(0)=-\frac{1}{2}$, these examples completely agree with the special cases $(3.6),(3.5)$, and (3.4), respectively, which have already been shown here to follow from our general series representations considered in Section 3.

\section{Acknowledgments}

The present investigation was supported, in part, by the Natural Sciences and Engineering Research Council of Canada under Grant OGP0007353.

\section{REFERENCES}

[1] V.S. Adamchik and H.M. Srivastava, Some series of the Zeta and related functions, Analysis 18(1998), 131-144.

[2] R. Apéry, Irrationalité de $\zeta(2)$ et $\zeta(3)$, in Journées Arithmétiques de Luminy (Colloq. Internat. CNRS, Centre Univ. Luminy, 1978), pp. 11-13, Astérisque 61, Soc. Math. France, Paris, 1979.

[3] R. Ayoub, Euler and the Zeta function, Amer. Math. Monthly 81(1974), 1067-1086.

[4] J.M. Borwein, D.M. Bradley, and R.E. Crandall, Computational strategies for the Riemann Zeta function, J. Comput. Appl. Math. (to appear).

[5] M.-P. Chen and H.M. Srivastava, Some families of series representations for the Riemann $\zeta(3)$, Resultate Math. 33(1998), 179-197.

[6] D. Cvijović and J. Klinowski, New rapidly convergent series representations for $\zeta(2 n+1)$, Proc. Amer. Math. Soc. 125(1997), 1263-1271. 
[7] A. Dąbrowski, A note on the values of the Riemann Zeta function at positive odd integers, Nieuw Arch. Wisk. (4) 14(1996), 199-207.

[8] J.A. Ewell, A new series representation for $\zeta(3)$, Amer. Math. Monthly 97(1990), 219-220.

[9] J.A. Ewell, On the Zeta function values $\zeta(2 k+1), k=1,2, \ldots$, Rocky Mountain J. Math. 25(1995), 1002-1012.

[10] M.L. Glasser, Some integrals of the arctangent function, Math. Comput. 22(1968), 445-447.

[11] R.W. Gosper, Jr., A calculus of series rearrangements, in Algorithms and Complexity: New Directions and Recent Results (J. F. Traub, Editor), pp. 121-151, Academic Press, New York and London, 1976.

[12] I.S. Gradshteyn and I.M. Ryzhik, Table of Integrals, Series, and Products (Corrected and Enlarged Edition Prepared by A. Jeffrey), Academic Press, New York, London, and Toronto, 1980.

[13] E.R. Hansen, A Table of Series and Products, Prentice-Hall, Englewood Cliffs, New Jersey, 1975.

[14] M.M. Hjortnaes, Overføring av rekken $\sum_{k=1}^{\infty}\left(1 / k^{3}\right)$ til et bestemt integral, Proceedings of the Twelfth Scandanavian Mathematical Congress (Lund; August 10-15, 1953), pp. 211-213, Scandanavian Mathematical Society, Lund, 1954.

[15] K.S. Kölbig, Nielsen's generalized Polylogarithms, SIAM J. Math. Anal. 17(1986), 1232-1258.

[16] L. Lewin, Polylogarithms and Associated Functions, Elsevier (North Holland) Science Publishers, New York, London, and Amsterdam, 1981.

[17] W. Magnus, F. Oberhettinger, and R.P. Soni, Formulas and Theorems for the Special Functions of Mathematical Physics, Die Grundlehren der Mathematischen Wissenschaften in Einzeldarstellungen mit besonderer Berücksichtingung der Anwendungsgebiete, Bd. 52, Springer-Verlag, New York, 1966.

[18] A.P. Prudnikov, Yu. A. Brychkov, and O.I. Marichev, Integrals and Series: Elementary Functions, "Nauka", Moscow, 1981 (in Russian).

[19] V. Ramaswami, Notes on Riemann's ל-function, J. London Math. Soc. 9(1934), 165-169.

[20] H.M. Srivastava, A unified presentation of certain classes of series of the Riemann Zeta function, Riv. Mat. Univ. Parma (4) 14(1988), 1-23.

[21] H.M. Srivastava, Further series representations for $\zeta(2 n+1)$, Appl. Math. Comput. 97(1998), 1-15.

[22] H.M. Srivastava, Some rapidly converging series for $\zeta(2 n+1)$, Proc. Amer. Math. Soc. 127(1999), 385-396.

[23] E.C. Titchmarsh, The Theory of the Riemann Zeta-Function, Oxford University (Clarendon) Press, Oxford and London, 1951; Second Edition (Revised by D.R. Heath-Brown), 1986.

[24] N.-Y. Zhang and K.S. Williams, Some series representations of $\zeta(2 n+1)$, Rocky Mountain J. Math. 23(1993), 1581-1592. 